\title{
Adaptive Network Security through Stream Machine Learning
}

\author{
Pavol Mulinka \\ CTU Czech Technical University in Prague \\ AIT Austrian Institute of Technology \\ pavol.mulinka@fel.cvut.cz
}

\author{
Pedro Casas \\ AIT Austrian Institute of Technology \\ pedro.casas@ait.ac.at
}

\begin{abstract}
Stream Machine Learning is rapidly gaining popularity within the network monitoring community as the big data produced by network devices and end-user terminals goes beyond the memory constraints of standard monitoring equipment. We consider a stream-based machine learning approach to network security, conceiving adaptive machine learning algorithms for the analysis of continuously evolving network data streams. Using a sliding-window adaptive-size approach, we show that adaptive random forests models are able to keep up with important concept drifts in the underlying network data streams, by keeping high accuracy with continuous re-training at concept drift detection times.
\end{abstract}

\section{CCS CONCEPTS}

- Computing methodologies $\rightarrow$ Machine learning; $\bullet$ Security and privacy $\rightarrow$ Network security;

\section{KEYWORDS}

Data Stream mining; Machine Learning; Network Attacks

\section{ACM Reference format:}

Pavol Mulinka and Pedro Casas. 2018. Adaptive Network Security through Stream Machine Learning. In Proceedings of PostersDemos '18: Posters and Demos, Budapest, Hungary, August 20-25, 2018 (Posters-Demos '18), 2 pages.

https://doi.org/10.1145/3234200.3234246

The research leading to these results has been partially funded by the Vienna Science and Technology Fund (WWTF) through project ICT15-129, "BigDAMA".

Permission to make digital or hard copies of all or part of this work for personal or classroom use is granted without fee provided that copies are not made or distributed for profit or commercial advantage and that copies bear this notice and the full citation on the first page. Copyrights for components of this work owned by others than ACM must be honored. Abstracting with credit is permitted. To copy otherwise, or republish, to post on servers or to redistribute to lists, requires prior specific permission and/or a fee. Request permissions from permissions@acm.org.

Posters-Demos '18, August 20-25, 2018, Budapest, Hungary

(c) 2018 Association for Computing Machinery.

ACM ISBN 978-1-4503-5915-3/18/08 .. \$15.00

https://doi.org/10.1145/3234200.3234246

\section{INTRODUCTION}

The application of machine learning models to network security and anomaly detection problems has largely increased in the last decade; however, the general approach in the literature still considers the analysis as an off-line learning problem, where models are trained once and then applied to the incoming measurements. We consider a stream-based approach for machine-learning-based network security; stream machine learning analysis consists of processing a data instance at a time, inspecting it only once, and as such, using a limited amount of memory. We evaluate the performance of two popular stream-based machine learning algorithms - Hoeffding Adaptive Trees (HAT) and Adaptive Random Forests (ARF), on the detection of different types of network attacks, using real network measurements.

We implement these models using an adaptive size windowbased approach known as ADWIN (ADaptive WINdowing) [2]. In a nutshell, ADWIN maintains a window of variable size containing training samples. The algorithm automatically grows the window when no change is apparent, and shrinks it when the statistical properties of the stream changes. ADWIN automatically adjusts its window size to the optimum balance point between reaction time and small variance; using ADWIN, we evaluate the proposed algorithms following a new strategy to evaluate stream-based algorithms [3], using prequential $\mathrm{k}$-fold cross validation; the strategy is basically an adaptation of cross-validation to the distributed streaming setting. As evaluation metric, we simply take the model detection accuracy (ACC).

\section{STREAM MACHINE LEARNING}

The analysis of stream-based machine learning models on the detection of different types of network attacks is performed on real network traffic measurements collected at the WIDE backbone network, using the MAWILab dataset for attacks labeling [1]. MAWILab is a public collection of 15-minute network traffic traces captured every day on a backbone link between Japan and the US since 2001. Building on this repository, the MAWILab project uses a combination of four traditional anomaly detectors to partially label the collected traffic. A commonly applied approach for evaluating the performance of stream-based algorithms is to benchmark them 


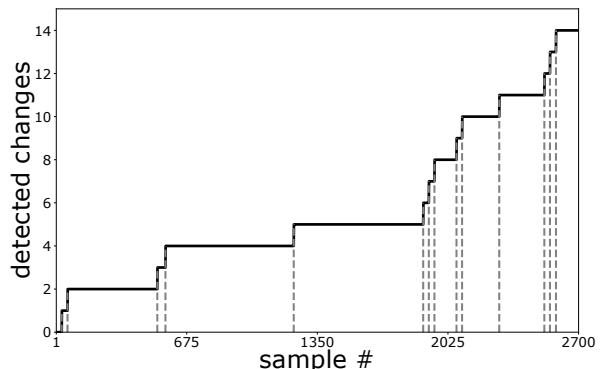

Figure 1: Page-Hinkley Concept Drift Detection.

against their corresponding off-line, batch implementations. Therefore, we use as baseline the results corresponding to batch-based algorithms, including Hoeffding Tree (HT-batch) and Random Forest (RF-batch). We subtract the batch metric results from the prequential results to show the performance of stream algorithms in comparison to their batch versions.

\subsection{Dataset description}

The traffic studied in this paper spans two months of packet traces collected in late 2015. From the labeled anomalies and attacks, we focus on a specific group which are detected simultaneously by the four MAWILab detectors, using in particular those events which are labeled as "anomalous" by MAWILab. We consider in particular 4 types of attacks/anomalies: (i) DDoS attacks (DDoS), (2) HTTP flashcrowds (mptp-la), (3) Flooding attacks (Ping flood), and (4) distributed network scans using UDP probing traffic (ntscUDP). We train the different models to detect each of these attack types separately, thus each detection approach consists of four different detectors, each of them specialized in detecting one of the aforementioned attacks types.

To perform the analysis in s stream-based manner, we split the traffic traces in consecutive time slots of five seconds each, and compute a set of features describing the traffic in each of these slots. In addition, each slot $i$ is assigned a label $l_{i}$, consisting of a binary vector $l_{i} \in \mathbb{R}^{4 \times 1}$ which indicates at each position if anomaly of type $j=1 . .4$ is present or not in current time slot. We compute a large number $n=245$ features describing a time slot $i=1 . . m$, using traditional packet measurements including traffic throughput, packet sizes, IP addresses and ports, transport protocols, flags, etc.

An important element to deal with when working with stream machine learning is the so-called concept drift; it happens when the statistical properties of the analyzed dataset abruptly shift in time. To detect concept drifts, we apply a standard statistical test for change detection known as the Page-Hinkley test, commonly used for change detection in time series analysis. Fig. 1 depicts the cumulative number of changes observed in the dataset, as well as the times when those changes are detected. Concept drifts permit to better
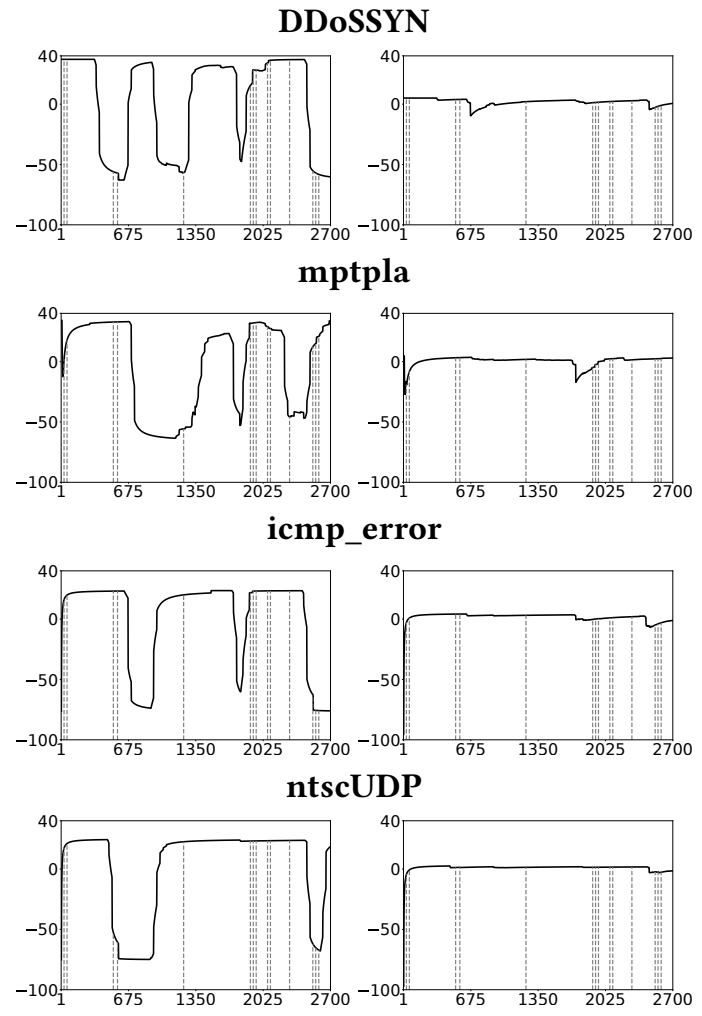

Figure 2: Prequential 10-fold cross evaluation. Concept drifts are marked with dashed lines.

understand when and why the window size of the ADWIN algorithm changes along time.

We evaluate the performance of the algorithms in four binary classification variants - one for each attack type, resulting in four sets of results for each tested algorithms. Fig. 2 reports the performance results for each attack type, considering detection accuracy as performance metric, and using the batch-algorithms accuracy as baseline. The prequential 10 -fold CV performance evaluation shows that the Adaptive Random Forests (ARF) model rapidly converges to the batch-based accuracy results, with minimum performance variations under concept drifts. On the other hand, the HAT model does not show any apparent convergence and results tend to oscillate around the batch algorithm baseline. In the case of HAT we can appreciate the correlation between the detected concept drifts and the performance variations of the model.

\section{REFERENCES}

[1] R. Fontugne et al., "Mawilab: combining diverse anomaly detectors for automated anomaly labeling and performance benchmarking," in Proc. ACM CoNEXT Conference, 2010.

[2] A. Bifet and R. Gavalda, "Learning from time-changing data with adaptive windowing," in Proc. SIAM Conference, 2007.

[3] A. Bifet et al., "Efficient online evaluation of big data stream classifiers," in Proc. ACM SIGKDD Conference, 2015. 